\title{
Influência ambiental sobre características de desempenho pré-desmama de bovinos Devon no Rio Grande do Sul ${ }^{1}$
}

\author{
Marcela Bicca Bragança Corrêa², Nelson José Laurino Dionello ${ }^{3}$, Fernando Flores Cardoso 4 \\ ${ }^{1}$ Parte da dissertação apresentada pela primeira autora à FAEM/UFPEL para obtenção do título de Mestre em Ciências. Financiada pela \\ CAPES. \\ ${ }^{2}$ Mestranda do Departamento de Zootecnia/FAEM/UFPEL. Bolsista CAPES. \\ ${ }^{3}$ Departamento de Zootecnia/FAEM/UFPEL. \\ ${ }^{4}$ Embrapa Pecuária Sul.
}

\begin{abstract}
RESUMO - O objetivo neste estudo foi avaliar o efeito dos fatores ambientais, do grupo de contemporâneos (GC), da data juliana do nascimento (DJN), da idade da vaca ao parto (IV) e da idade do bezerro à desmama (IT) sobre características de desempenho produtivo na fase pré-desmama de bovinos Devon criados no Rio Grande do Sul. Foram analisados o peso ao nascer (PN) e à desmama (PD) e o ganho do nascimento à desmama ajustado para 205 dias (GNDA). Foram utilizados registros de 23.369 animais, coletados pelo PROMEBO - Programa de Melhoramento de Bovinos de Carne, conduzido pela Associação Nacional de Criadores - "Herd Book Collares", nascidos entre 1980 e 2000. Destes animais, 16.593 tinham registro de PN, 22.530 tinham de PD e 22.259 de GNDA. As análises foram realizadas pelo PROC MIXED do SAS, cujo modelo considerou, além dos efeitos ambientais citados, o efeito aleatório do touro e o erro residual. A DJN foi considerada pela freqüência de nascimento dentro de classes de dez dias, em um total de 13 classes. A primeira, iniciando aos 190 dias do ano até a classe 13, dos 310 aos 320 dias. Para PN, os animais nascidos na classe 12 (out-nov) foram 3,15\% mais pesados que os da classe 190 (jul), ao contrário do PD e GNDA, em que os melhores resultados foram na classe 190, respectivamente, 11,51 e $9,99 \%$ superiores aos nascidos na classe 13 (nov). O efeito da idade da vaca apresentou ponto de máxima em torno dos oito anos de idade, demonstrando tendência quadrática. O efeito da idade do bezerro apresentou tendência quadrática para GNDA e linear para PD. Esses resultados indicam que a inclusão dos efeitos ambientais no modelo de análise das características produtivas pré-desmama é necessária para que as avaliações genéticas sejam adequadamente ajustadas e, portanto, mais precisas e confiáveis.
\end{abstract}

Palavras-chave: bovinos de corte, data juliana do nascimento, ganho de peso pré-desmama, grupo de contemporâneos, pesos

\section{Environmental effects on pre-weaning performance traits of Devon cattle in Rio Grande do Sul}

\begin{abstract}
The objective of this study was to evaluate the environmental effects of contemporary groups (CG), Julian birth date (JBD), age of dam at calving (AD) and age of calf at weaning (AC) on pre-weaning performance traits: birth weight (BW), weaning weight (WW) and 205 days adjusted weaning gain (WG) of Devon cattle raised in Rio Grande do Sul. A total of 23,369 records on animals, born from 1980 to 2000, collected by PROMEBO - beef cattle improvement program of Associação Nacional de Criadores Herd Book Collares were used. Analyses were performed by PROC MIXED of SAS, using a model that included $\mathrm{CG}, \mathrm{JBD}$ and $\mathrm{AD}$ as fixed effects and sire and residual error as random effects. Average BW of animals that were born in Oct-Nov was 3.15\% higher than BW of animals that were born in Jul, which were respectively for WW and WG $11.51 \%$ and $9.99 \%$ heavier than animals that were born in Nov. Age of dam showed a quadratic effect with maximal response on all traits around eight years,. The age of calf effect was quadratic on WG and linear on WW. These results indicate environmental effects should be included in models used for genetic evaluations of pre-weaning performance traits in order to improve their precision and reliability.
\end{abstract}

Key Words: beef cattle, contemporary groups, Julian birth date, pre-weaning weight gain, weights

\section{Introdução}

A produção de carne é uma atividade econômica de grande importância no Brasil, sendo reconhecida pela qualidade e pelo volume de produção. Atualmente, o país é o maior exportador mundial de carne, incluindo a carne bovina, que teve aumento na exportação de 464 para 1.350 mil toneladas métricas, equivalentes ao peso da carcaça dos anos de 1999 a 2004. Ou seja, as exportações de carne bovina praticamente triplicaram em cinco anos (Brasil - MAPA, 2004). 
A competitividade do mercado e a crescente exigência do mercado consumidor, interno e externo, por um produto de qualidade fazem com que a cadeia produtiva se torne mais eficiente economicamente, melhorando os índices de produtividade e a qualidade dos produtos.

Uma das ferramentas para se incrementar o desempenho produtivo nos rebanhos é o melhoramento genético animal, por meio da seleção de animais geneticamente superiores, visando ganho genético nas futuras gerações, em decorrência da maior freqüência de alelos favoráveis às características economicamente importantes, aumentando a quantidade e melhorando a qualidade do produto.

Segundo FerrazFilho et al. (2002), características de crescimento, como peso corporal, medidas na fase inicial do desenvolvimento do animal, são importantes na determinação da eficiência econômica de qualquer sistema de produção de bovinos e podem ser recomendadas como critério de seleção.

O efeito da idade da mãe sobre o desenvolvimento do bezerro, expresso pelo ganho de peso, varia em função das diferenças permanentes entre vacas, dos períodos de gestação e aleitamento, do ambiente uterino, da produção de leite e da habilidade materna e, inclusive, da imunidade passiva obtida pelo colostro (Koch, 1972).

A idade do bezerro também apresenta um efeito que deve ser considerado nas avaliações genéticas. Conforme Pelicioni et al. (2002), a idade do bezerro à desmama tem grande influência sobre o peso à desmama e o ganho de peso no período do nascimento à desmama, observando-se decréscimo do ganho à medida que aumenta a idade do animal.

$\mathrm{Na}$ fase pré-desmama, são observados os maiores ganhos de peso em condições extensivas de criação. A identificação e seleção de animais de crescimento acelerado nesta fase têm sido usadas pelos produtores com o objetivo de melhorar a eficiência dos sistemas de criação. Neste contexto, o peso ao nascer também tem sido considerado, por ser utilizado no cálculo dos ganhos de peso ajustados e por ser um importante critério de seleção para evitar os problemas de parto, especialmente em novilhas (Cardoso, 1999).

A raça Devon é bastante criada no Rio Grande do Sul, onde se encontram os maiores rebanhos dessa raça no mundo. Embora os animais apresentem precocidade média e, entre as raças britânicas criadas no Rio Grande do Sul, a Devon seja a mais tardia, as novilhas podem ser colocadas em reprodução uma idade jovem. Quanto à rusticidade, é a mais rústica das raças britânicas, sendo menos exigente com relação à qualidade do alimento. As fêmeas, quando adequadamente manejadas, podem apresentar elevados índices de concepção e parição, apresentando boa habilidade materna, com produção de leite que cria bem o bezerro. Os novilhos apresentam carcaças pesadas e com boa quantidade de porção comestível de qualidade, com boa gordura de marmorização. Além disso, são animais de temperamento dócil (Jardim \& Pimentel, 1985).

O objetivo neste estudo foi avaliar a influência ambiental (grupo de contemporâneos, data juliana de nascimento, idade da vaca e idade do bezerro à desmama) sobre a produção de carne, expressa pelo peso ao nascer, pelo ganho de peso do nascimento à desmama e pelo peso à desmama, no rebanho da raça Devon no Rio Grande do Sul.

\section{Material e Métodos}

O estudo foi desenvolvido utilizando-se dados coletados rotineiramente pelo PROMEBO - Programa de Melhoramento de Bovinos de Carne, conduzido pela Associação Nacional de Criadores - "Herd Book Collares" dos animais da raça Devon que participaram do programa nos anos de 1980 a 2000. Foram utilizados dados de 23.369 animais, dos quais 16.593 tinham registro de peso ao nascer (PN), 22.530 apresentavam dados de peso à desmama (PD) e 22.259 tinham informações de ganho do nascimento à desmama ajustado para 205 dias (GNDA).

$\mathrm{O} P \mathrm{~N}$ foi obtido por pesagem nas primeiras 24 horas de vida do animal, no momento das anotações do nascimento, com balança a campo. O PD foi obtido no momento da desmama dos animais, com jejum prévio de 12 horas, com os animais separados das mães, para evitar distorções decorrentes da ingestão de líquidos. A partir dos dois pesos, foi obtido o GNDA, calculado pela seguinte equação: GNDA $=((\mathrm{PD}-\mathrm{PN}) / \mathrm{IT}) \times 205$, em que IT corresponde à idade do bezerro, em dias, no momento da desmama.

Os efeitos fixos e classificatórios do modelo foram os grupos de contemporâneos e a data juliana do nascimento.

Os grupos de contemporâneos foram formados incluindo criador-rebanho, ano da produção (safra), estação de produção, sexo, grupo de manejo (alimentar) e data da pesagem de desmama. Grupo de contemporâneos com apenas um animal e touros com somente um filho foram previamente excluídos da análise, pois não fornecem informações relevantes para comparações.

O efeito da data juliana do nascimento foi considerado no modelo com base em classes de dez dias cada uma, em um total de 13 classes: a primeira dos 190 aos 200 dias do ano (meados de julho) e a classe 13 dos 310 aos 320 dias (meados de novembro). Portanto, todos os nascimentos ocorreram dentro deste período de 130 dias (10/07 a 17/11). 
A idade da vaca ao parto, variando de 2 a 14 anos de idade, foi considerada covariável, com coeficiente de regressão linear e quadrático.

A idade do bezerro à desmama também foi considerada covariável, com coeficientes de regressão linear e quadrático. O GNDA é ajustado para 205 dias de idade, mas houve variação de 200 dias entre os animais, o que pode causar desvio da linearidade do efeito.

Os dados foram analisados utilizando-se o programa Statistical Analysis System (SAS, 1996), por meio do PROC MIXED, e os testes de efeitos fixos do modelo foram realizados pelo teste F tipo III:

$$
\mathrm{Y}_{\mathrm{ijkm}}=\mu+\mathrm{GC}_{\mathrm{i}}+\mathrm{DJN}_{\mathrm{j}}+\mathrm{IV}+\mathrm{IV}^{2}+\mathrm{IT}+\mathrm{IT}^{2}+\mathrm{T}_{\mathrm{k}}+\mathrm{e}_{\mathrm{ijkm}}
$$

em que: $\mathrm{Y}_{\mathrm{ijkm}}=$ valor observado (PN, PD, GNDA); $\mu=$ média geral; $\mathrm{GC}_{\mathrm{i}}=$ efeito do grupo de contemporâneos; $\mathrm{DJN}_{\mathrm{j}}=$ efeito da data juliana de nascimento; IV = coeficiente de regressão linear para idade da vaca; $I^{2}=$ coeficiente de regressão quadrático para idade da vaca; IT = coeficiente de regressão linear para idade do bezerro; IT $^{2}=$ coeficiente de regressão quadrático para idade do bezerro; $\mathrm{T}_{\mathrm{k}}=$ efeito aleatório do touro; e $\mathrm{e}_{\mathrm{ijkm}}=$ erro residual.

Para a análise do PN, foi excluído do modelo o efeito da idade do bezerro à desmama.

\section{Resultados e Discussão}

As médias para as características estudadas são apresentadas na Tabela 1. A média observada para PN de $34,32 \mathrm{~kg}$ foi inferior às encontradas por Fernandes et al. (2002) e Boligon et al. (2004), de 41,55 e 41,63 kg, respectivamente, para animais da raça Charolês, uma raça de maior porte, no Rio Grande do Sul. Ferreira et al. (1999) encontraram média de $35,9 \mathrm{~kg}$ para o PN de bovinos Hereford no mesmo estado. Porém, a média encontrada neste estudo foi superior à obtida em outros trabalhos com dados de bovinos, como a encontrada por Cardoso et al. (2001), de 32,87 kg para a raça Aberdeen Angus, mas muito semelhante à encontrada por Cundiff et al. (1995) na raça Devon, de $34 \mathrm{~kg}$, ou seja, a média observada está de acordo com o padrão para o PN de bovinos Devon, que, segundo Jardim \& Pimentel (1995), é de $34 \mathrm{~kg}$.

Para a característica GNDA, a média foi de $138,20 \mathrm{~kg}$, abaixo do ganho encontrado por Dal-Farra et al. (2002), em bovinos cruzas Nelore x Angus e Nelore x Hereford, que foi de $148,49 \mathrm{~kg}$, provavelmente pela heterose e complementaridade dos produtos que os autores obtiveram pelo cruzamento. Fernandes et al. (2002) encontraram média de
Tabela 1 - Médias, desvios-padrão (DP), coeficientes de variação (CV) e amplitudes observadas para peso ao nascer (PN), ganho do nascimento à desmama ajustado para 205 dias (GNDA), peso à desmama (PD) e idade do bezerro à desmama (IT)

Table 1 - Means, standard deviations (SD), coefficients of variation (CV) and range for birth weight (BW), 205 day adjusted weaning gain (WG), weaning weight (WW) and age of calf at weaning $(A W)$

\begin{tabular}{|c|c|c|c|c|c|}
\hline $\begin{array}{l}\text { Carácter } \\
\text { Trait }\end{array}$ & $\begin{array}{l}\text { Média } \\
\text { Mean }\end{array}$ & $\begin{array}{l}\mathrm{DP} \\
S D\end{array}$ & $\begin{array}{l}\mathrm{CV}(\%) \\
\mathrm{CV}(\%)\end{array}$ & $\begin{array}{l}\text { Mínimo } \\
\text { Minimum }\end{array}$ & $\begin{array}{l}\text { Máximo } \\
\text { Maximum }\end{array}$ \\
\hline $\begin{array}{l}\text { Peso ao nascer }(\mathrm{kg}) \\
\text { Birth weight }(\mathrm{kg})\end{array}$ & 34,32 & 2,94 & 8,5 & 20,00 & 45,00 \\
\hline $\begin{array}{l}\text { Ganho de peso } \\
\text { nascimento à desmama } \\
\text { ajustado para } 205 \text { dias } \\
205 \text { day adjusted } \\
\text { weaning gain }(\mathrm{kg})\end{array}$ & 138,20 & 34,31 & 24,8 & 41,66 & 229,90 \\
\hline $\begin{array}{l}\text { Peso à desmama }(\mathrm{kg}) \\
\text { Weaning weight }(\mathrm{kg})\end{array}$ & 157,06 & 34,60 & 22 & 80,00 & 260,00 \\
\hline $\begin{array}{l}\text { Idade do bezerro (dias) } \\
\text { Age at weaning (days) }\end{array}$ & 194,88 & 34,65 & 18 & 100,00 & 300,00 \\
\hline
\end{tabular}

154,79 de ganho de peso pré-desmama para bovinos Charolês e Mello (1999) obteve, para a raça Canchim, 154,34 $\mathrm{kg}$ de ganho, enquanto Ferreira et al. (1999) relataram média de $166,6 \mathrm{~kg}$ para animais da raça Hereford, sendo estes três últimos estudos realizados no Rio Grande do Sul. Cardoso (1999) obteve média de 124,09 kg de ganho de peso prédesmame em bovinos Angus criados no mesmo estado, semelhante às obtidas nos demais estudos realizados com bovinos da raça Hereford no Sul do Brasil (Cardelino \& Cardelino, 1984; Fries, 1984; Pons et al., 1989). Porém, os resultados obtidos neste estudo foram semelhantes à média encontrada por Paz et al. (1999), que obtiveram 0,681 kg/dia, o que, em 205 dias, resultaria em 139,60 kg para bovinos Nelore. A literatura, entretanto, carece de trabalhos utilizando a raça Devon, o que dificulta discussões e comparações sobre o desempenho dos indivíduos estudados em outras populações da mesma raça.

Para a característica PD, a média observada de $157,06 \mathrm{~kg}$ está abaixo do peso encontrado em outros estudos com bovinos de corte (Ferraz Filho et al., 2002), em que se obteve média de 170,30 kg para animais da raça Nelore em várias regiões do Brasil (Fridrich et al., 2004), resultando em média de 167,83 kg para o PD de bovinos Tabapuã na Região Sul do Brasil. Boligon et al. (2004) encontraram média de $185,38 \mathrm{~kg}$ para o PD de bovinos Charolês no Rio Grande do Sul. Contudo, as raças das demais pesquisas são de porte maior que a Devon.

Na Tabela 2 consta o resumo dos testes de significância para os efeitos fixos do modelo sobre PN, GNDA e PD. Os efeitos ambientais testados foram altamente significativos 
Tabela 2 - Teste F tipo III e probabilidade de significância para as fontes de variação do modelo de peso ao nascer (PN), ganho do nascimento à desmama ajustado para 205 dias (GNDA) e peso à desmama (PD)

Table 2 - $\quad$ Type III F test and significance level for effects included in models for birth weight (BW), 205 day adjusted weaning gain (WG) and weaning weight $(W W)$

\begin{tabular}{|c|c|c|c|c|c|c|c|c|c|}
\hline \multirow[b]{2}{*}{$\begin{array}{l}\text { Efeitos } \\
\text { Effects }\end{array}$} & \multicolumn{3}{|c|}{$\mathrm{PN}(B W)$} & \multicolumn{3}{|c|}{ GNDA $(W G)$} & \multicolumn{3}{|c|}{$\mathrm{PD}(W W)$} \\
\hline & G. $\mathrm{L}^{1}$ & F & $\operatorname{Pr}>F$ & G.L & F & $\operatorname{Pr}>F$ & G.L & F & $\operatorname{Pr}>\mathrm{F}$ \\
\hline $\mathrm{GC}^{2}$ & 629 & 18,04 & $<.0001$ & 909 & 16,49 & $<.0001$ & 912 & 14,26 & $<.0001$ \\
\hline $\mathrm{DJN}^{3}$ & 12 & 6,87 & $<.0001$ & 12 & 11,85 & $<.0001$ & 12 & 19,05 & $<.0001$ \\
\hline $\mathrm{IV}^{4}$ & 1 & 68,79 & $<.0001$ & 1 & 797,66 & $<.0001$ & 1 & 866,86 & $<.0001$ \\
\hline $\mathrm{IV}^{2,} 5$ & 1 & 50,85 & $<.0001$ & 1 & 613,11 & $<.0001$ & 1 & 671,67 & $<.0001$ \\
\hline $\mathrm{IT}^{6}$ & 1 & 78,44 & $<.0001$ & 1 & 100 & $<.0001$ & & & \\
\hline $\mathrm{IT}^{2,} 7$ & 1 & 24,95 & $<.0001$ & 1 & 3,33 & 0.6064 & & & \\
\hline
\end{tabular}

1 Graus de liberdade (degrees of freedom).

2 Grupos de contemporâneos (contemporary groups).

3 Data juliana do nascimento (julian birth day).

4 Idade da vaca - linear (age of dam - linear).

5 Idade da vaca - quadrático (age of dam - quadratic).

6 Idade do bezerro à desmama - linear (age of calf at weaning - linear).

7 Idade do bezerro à desmama - quadrático (age of calf at weaning-quadratic)

para as três características estudadas, exceto para $\mathrm{PD}$, na qual o coeficiente de regressão quadrático da idade do bezerro à desmama não apresentou significância, evidenciando tendência linear.

As médias ajustadas e os erros-padrão para PN, GNDA e PD, pela classe da data juliana do nascimento (DJN), são apresentadas nas Figuras 1, 2 e 3, respectivamente.

O PN foi menor para os animais nascidos na classe 190 (jul), 34,04 kg, apresentando tendência crescente até a classe 300 (out-nov), $35,26 \mathrm{~kg}$, demonstrando superioridade de $1,22 \mathrm{~kg}$, equivalente a $3,15 \%$, em função da época do nascimento. Ou seja, os animais que nasceram em datas mais próximas à primavera, quando há maior disponibilidade de forragem, apresentaram maiores pesos ao nascer, pois suas mães apresentavam melhor condição corporal no terço final da gestação e, conseqüentemente, ao parto. Cardoso

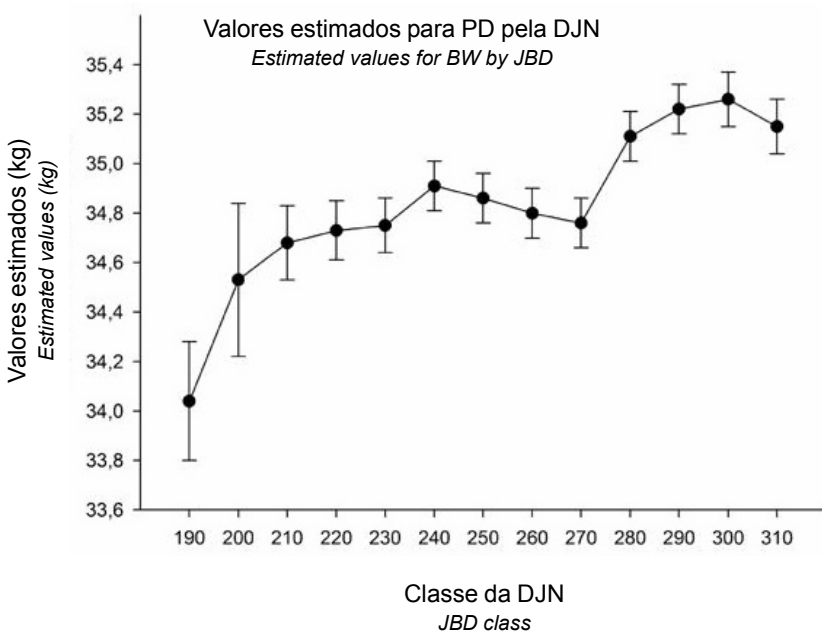

Figura 1 - Médias ajustadas e erros-padrão de PN pela classe da DJN.

Figure 1 - BW adjusted means and standard error by JBD class.

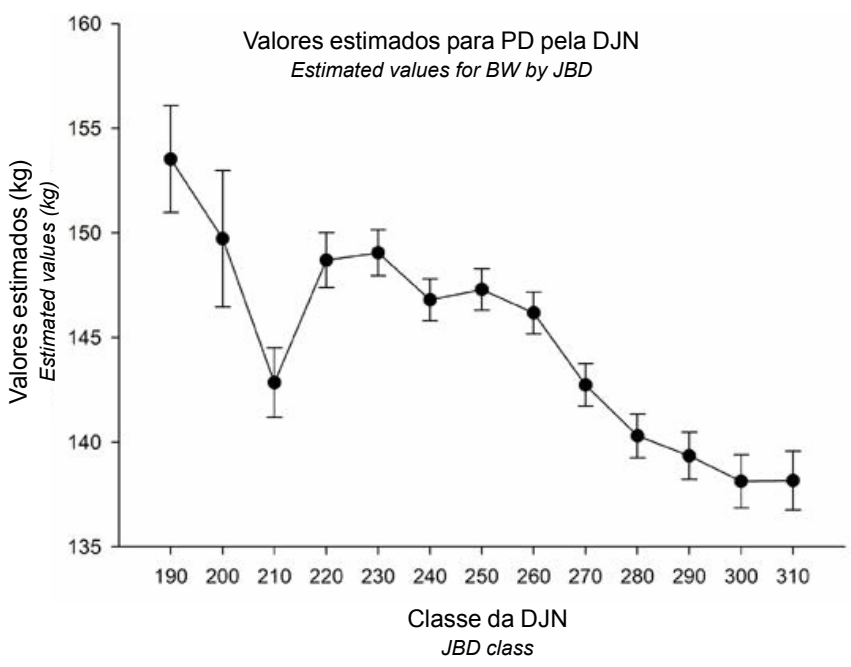

Figura 2 - Médias ajustadas e erros-padrão de GNDA pela classe da DJN.

Figura 2 - WG adjusted means and standard error by JBD class.

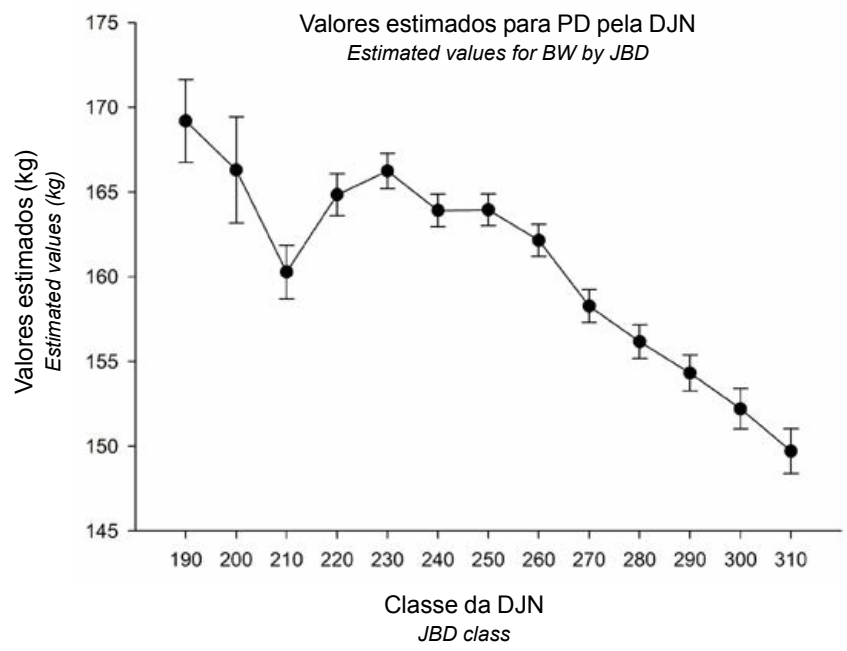

Figura 3 - Médias ajustadas e erros-padrão de PD pela classe da DJN.

Figure 3 - $\quad$ WW adjusted means and standard error by JBD class. 
(1999) também encontrou maiores pesos para os bezerros nascidos na primavera, nesse caso, em relação aos nascidos no outono, na raça Angus. Por outro lado, vários autores (Barcellos \& Lobato, 1992; Eler et al., 1989; Milagres et al. (1993), com bovinos cruzas Hereford x Nelore e Hereford, obtiveram PN maior para os animais nascidos no outono.

Para GNDA, os melhores resultados foram obtidos para os animais da classe 190 (jul.), sendo $15,35 \mathrm{~kg}$ acima dos animais nascidos na classe 300 (out-nov). Esses resultados corroboram o encontrado por Pelicioni et al. (2002), em que animais nascidos no início da primavera apresentaram maiores ganhos de peso, justificados pelo fato de as mães estarem bem alimentadas e, portanto com maior produção de leite e disponibilidade de alimento para suas crias. Dal-Farra et al. (2002) também verificaram maiores ganhos de peso nos animais nascidos no início da primavera, para bovinos cruzas Nelore x Angus, Nelore x Hereford. Além destes, vários autores obtiveram resultados semelhantes (Campos et al., 1989; Barcellos \& Lobato, 1992; Teixeira, 2000), evidenciando (Fries (1996) que a adoção de uma estação de monta que resulte em nascimentos no início da primavera proporciona os melhores resultados.

A influência da DJN sobre o PD, assim como sobre o GNDA, promoveu melhores pesos para os nascidos na primeira classe (jul), sendo $169,19 \mathrm{~kg}$, com tendência decrescente até a classe 13 (nov), apresentando 149,71 kg, ou seja, 19,48 kg a menos. Os animais nascidos em julho, quando aprendem a pastejar, ou seja, quando não dependem exclusivamente da mãe, por volta dos três meses, encontram maior e melhor disponibilidade de forragem e, portanto, são favorecidos quanto aos pesos. Esta relação obtida em função da DJN é contrária à encontrada por Pelicioni etal. (2002), que obtiveram maiores pesos à desmama para animais nascidos entre 265 e 310 dias.

Pode-se observar pelas figuras que o ganho de peso (GNDA) e o peso à desmama (PD) comportaram-se da mesma forma em relação à época do ano, enquanto o peso ao nascer $(\mathrm{PN})$ apresentou pequena variação. Para as três características estudadas, o teste de médias Tukey $(a=0,05)$, realizado entre as 13 classes, resultou em diferenças estatísticas significativas.

Os parâmetros das equações de predição para $\mathrm{PN}$, GNDA e PD, em função da data juliana do nascimento, da idade da vaca ao parto e da idade do bezerro à desmama são descritos na Tabela 3 .

O efeito da idade da vaca (IV) sobre as características estudadas foi significativo, com tendência quadrática para PN, GNDA e PD. A IV apresentou ponto de máxima médio, para as três características, aos 7,89 anos. Portanto, as vacas em maturidade fisiológica para habilidade materna (produção de leite) desmamaram bezerros mais pesados que as vacas jovens ou que ultrapassaram esta fase. Este resultado corrobora os de vários autores que consideram esse efeito, como Dal-Farra et al. (2002), que encontraram efeito significativo para as características de ganho do nascimento à desmama e escores de conformação, precocidade e musculatura, demonstrando que o desempenho dos bezerros melhorou com o aumento da idade das vacas cruzas com diversos graus de sangue Nelore, atingindo o pico por volta dos sete anos, que decresceu gradativamente a partir desta idade. Pelicioni et. al (2002) também obtiveram resultados semelhantes para a raça Gir, de modo que,

Tabela 3 - Parâmetros das equações de regressão para peso ao nascer (PN), ganho de peso do nascimento à desmama (GNDA) e peso à desmama (PD), em função da data juliana do nascimento (DJN), da idade da vaca (IV) e da idade do bezerro à desmama (IT)

Table 3 - $\quad$ Regression parameters for birth weight (BW), weaning gain (WG) and weaning weight (WW) on Julian birth date (JBD), age of dam (AD) and age of calf at weaning $(A C)$

\begin{tabular}{lccc}
\hline & & \multicolumn{2}{c}{$\begin{array}{c}\text { Parâmetro } \\
\text { Parameter }\end{array}$} \\
\cline { 3 - 3 } $\begin{array}{l}\text { Carácter } \\
\text { Trait }\end{array}$ & $\begin{array}{c}\text { Intercepto } \\
\text { Intercept }\end{array}$ & $\mathrm{b} 1$ & $\mathrm{~b} 2$ \\
\hline DJN $(J D B)$ & & & $\mathrm{r}^{2}$ \\
PN $(B W)$ & 31,92 & 0,0193 & $-0,0004$ \\
GNDA $(W G)$ & 146,43 & 0,1033 & $-0,0012$ \\
PD $(W W)$ & 123,24 & 0,4487 & 0,77 \\
Idade da vaca $(A D)$ & & & $-0,02068$ \\
PN $(B W)$ & 34,89 & 0,3314 & $-0,6834$ \\
GNDA $(W G)$ & 171,77 & 10,7172 & $-0,6698$ \\
PD $(W W)$ & 10,48 & 10,4730 & \\
Idade do bezerro $(A C)$ & & & 0,8900723 \\
GNDA $(W G)$ & 171,77 & $-0,4924$ & $-0,00025$ \\
PD $(W W)$ & 10,47 & 0,5215 & \\
\hline
\end{tabular}


a partir dos quatro anos de idade da vaca, os bezerros mostraram tendências crescentes de peso à desmama e ganho médio diário à medida que a mãe atingiu a maturidade, e, após apresentarem um platô, o desempenho passou a declinar com o envelhecimento da vaca. Cardoso et al. (2001) também mostraram resultados compatíveis para vacas Angus ao relatarem que o desempenho materno máximo para peso ao nascer e ganho de peso do nascimento à desmama foi alcançado por vacas de oito anos de idade. Paz et al. (1999) demonstraram que as vacas Nelore atingiram a maturidade fisiológica de habilidade materna entre os cinco e sete anos de idade para o ganho médio diário até a desmama.

Além dos autores citados, Cardellino \& Castro (1987), em vacas Nelore, encontraram efeito quadrático para idade da vaca sobre o peso ao desmame e o ganho de peso pré desmame, que aumentaram até 6-9 anos, ocorrendo declínio após esta idade. Também Fries (1984), em vacas Hereford, e Campos et al. (1989), em vacas Nelore, utilizando polinômios segmentados, observaram sistema quadráticoquadrático para o efeito da idade da vaca sobre o ganho de peso pré-desmame. Porém, os resultados obtidos neste estudo diferem dos descritos por Martins et al. (2000), que não encontraram efeito significativo $(\mathrm{P}>0,05)$ da idade da vaca ao parto para as características de peso ao nascer, ganho de peso do nascimento à desmama e peso à desmama, em bovinos da raça Nelore.

$\mathrm{O}$ efeito da idade do bezerro à desmama apresentou significância tanto no coeficiente linear quanto no quadrático para GNDA e apenas para o coeficiente de regressão linear para PD. O GNDA apresentou declínio à medida que aumentou a idade do bezerro, como observado por Paz et al. (1999), que obtiveram menores ganhos médios de peso à medida que a idade do animal aumentou, e por Fries (1984) e Wolf (1996), que demonstraram a nãolinearidade do efeito da idade do bezerro ao desmame sobre o ganho de peso pré-desmame e o peso à desmama. Cardoso (1999) verificou, para animais mais jovens, resultados mais favoráveis de ganho de peso pré-desmama em relação aos mais velhos, tanto no outono quanto na primavera, demonstrando que o simples ajuste linear padrão para os 205 dias não é suficiente para remover as variações deste efeito.

\section{Conclusões}

A inclusão dos efeitos ambientais no modelo de análise das características de desempenho produtivo na fase prédesmama é necessária para que as avaliações genéticas sejam adequadamente ajustadas e, conseqüentemente, mais precisas e confiáveis.
Os efeitos de grupo de contemporâneos e da data juliana do nascimento são de extrema importância para a correta avaliação dos desempenhos, uma vez que a sazonalidade das pastagens naturais, típica do Sul do Brasil, afeta a produção nos meses críticos, podendo contribuir para a seleção equivocada dos rebanhos.

A utilização das covariáveis idade da vaca ao parto e idade do bezerro à desmama também promoveu benefícios às análises, pois, na fase pré-desmama, os animais dependem fundamentalmente de suas mães e, portanto, têm influência marcante nos seus desempenhos conforme a condição fisiológica da mãe. Além disso, o ajuste para idade padrão de 205 dias não remove o efeito da idade do bezerro no ganho pré-desmama.

\section{Literatura Citada}

BARCELOS, J.O.J.; LOBATO, J.F. P. Efeitos da época do nascimento no desenvolvimento de becerros Hereford e suas cruzas. I. Peso ao nascer e ganho médio diário pré-desmama. Revista Brasileira de Zootecnia, v.21, n.1, p.137-149, 1992.

BRASIL - MINISTÉRIO DA AGRICULTURA, PECUÁRIA E ABASTECIMENTO - MAPA. Disponível em: www.agricultura.gov.br Acesso em: 10/11/2004.

BOLIGON, A.A.; WEBER, T.; KIPPERT, C.J. et al. Componentes de variância e parâmetros genéticos para características de crescimento para a raça Charolês no estado do Rio Grande do Sul. In: REUNIÃO ANUAL DA SOCIEDADE BRASILEIRA DE ZootecniA, 41., 2004, Campo Grande. Anais... Campo Grande: Sociedade Brasileira de Zootecnia/V2 Comunicação, 2004 (CD-ROM). Melhoramento Animal. MELH 015.

CAMPOS, L.T.; SILVA, P.R.; FRIES, L.A. Fatores de correção para efeitos ambientais que afetam o ganho do nascimento à desmama em bovinos da raça Nelore. Uberaba: Empresa de Pesquisa Agropecuária de Minas Gerais, 1989. p.108-123 (Coletânea de Pesquisas inéditas sobre Zebu).

CARDELLINO, M.V.; CARDELLINO, R.A. Efeitos ambientais sobre o peso, ganho de peso e conformação à desmama em bovinos Hereford no Rio Grande do Sul. Revista Brasileira de Zootecnia, v.13, n.4, p.547-556, 1984.

CARDELINO, R.A.; CASTRO, L.F.S. Efeito ambientais e genéticos e fatores de correção para peso ao nascer, peso à desmama e ganho de peso pré-desmama, em bovinos Nelore. Revista Brasileira de Zootecnia, v.16, n.1, p.14-27, 1987.

CARDOSO, F.F. Caracterização genética do desempenho do nascimento à desmama de bovinos Aberdeen Angus criados no Rio Grande do Sul. Pelotas: Universidade Federal de Pelotas, 1999. 116p. Dissertação (Mestrado em Ciências) Universidade Federal de Pelotas, 1999.

CARDOSO, F.F.; CARDELlinO, R.A.; CAMPOS, L.T. Fatores ambientais que afetam o desempenho de nascimento à desmama de bezerros Angus criados no Rio Grande do Sul. Revista Brasileira de Zootecnia, v.30, n.2, p.326-335, 2001.

CUNDIFF, L.V. et al. [1995] Disponível em: www.herdbook.org.br/ clay centerdevon.htm $<$ Acesso em: 10/11/2004.

DAL-FARRA, R.A.; ROSO, V.M.; SCHENKEL, F.S. Efeitos de ambiente e de heterose sobre o ganho de peso do nascimento ao desmame e sobre os escores visuais ao desmame de bovinos de corte. Revista Brasileira de Zootecnia, v.31, n.3, p.1350 1361, 2002 (supl.).

ELER, J.P.; LOBO, R.B.; ROSA, A.N. Influência de fatores genéticos e de meio em pesos de bovinos da raça Nelore criados no estado 
de São Paulo. Revista Brasileira de Zootecnia, v.18, n.2, p.103-111, 1989.

FERNANDES, H.D.; FERREIRA, G.B.B.; RORATO, P.R.N. Tendências e parâmetros genéticos para características prédesmama em bovinos da raça Charolês criados no Rio Grande do Sul. Revista Brasileira de Zootecnia, v.31, n.1, p.321-330, 2002, 2002 (supl.).

FERRAZ FILHO, P.B.; RAMOS, A.A.; SILVA, L.O.C. et al. Tendência genética dos efeitos direto e materno sobre os pesos à desmama e pós-desmama de bovinos da raça Tabapuã no Brasil. Revista Brasileira de Zootecnia, v.31, n.2, p.635-640, 2002.

FERREIRA, G.B.; MacNEIL, M.D.; Van VLECK, L.D. Variance components and breeding values for growth traits from different statistical models. Journal of Animal Science, v.77, p.26412650,1999

FRIDRICH, A.B.; SILVA, M.A.; FERREIRA, I.C. et al. Estimativa de parâmetros genéticos dos pesos à desmama e a um ano de idade de bovinos da raça Tabapuã nas regiões brasileiras sul, sudeste, centro-oeste e nordeste. In: REUNIÃO ANUAL DA SOCIEDADE BRASILEIRA DE ZOOTECNIA, 41., 2004, Campo Grande. Anais ... Campo Grande: Sociedade Brasileira de Zootecnia/V2 Comunicação, 2004 (CD-ROM). Melhoramento Animal. MELH 011.

FRIES, L.A. A study of wearing weights in Hereford cattle in the state of Rio Grande do Sul, Brazil. Ames: Iowa State University, 1984. 317p. Thesis (Ph.D.) - Iowa State University, 1984.

FRIES, L.A. Efeito da data Juliana do nascimento obre o peso à desmama em zebuínos. In: SIMPÓSIO NACIONAL DE MELHORAMENTO GENÉTICO, 1., 1996, Ribeirão Preto. Anais... Ribeirão Preto: Sociedade Brasileira de Melhoramento Animal, 1996. p.249-251.

JARDIM, P.O.C.; PIMENTEL, M.A. Bovinos de corte. Pelotas: Editora Universitária, 1995. 125p.

$\mathrm{KOCH}, \mathrm{M} . \mathrm{R}$. The role of material effect in animal breeding. IV. Maternal effects in beef cattle. Journal of Animal Science, v.35, p.1316-1323, 1972.

MARTINS, G.A.; FILHO, R.M.; LIMA, F.A.M. et al. Influência de fatores genéticos e de meio sobre o crescimento de bovinos da raça Nelore no estado do Maranhão. Revista Brasileira de Zootecnia, v.29, n.1, p.103-107, 2000.
MELLO, S.P. Tendência genética para pesos em um rebanho da raça Canchim. Jaboticabal: Universidade Estadual Paulista, 1999. 78p. Dissertação (Mestrado em Zootecnia) - Universidade Estadual Paulista, 1999.

MILAGRES, J.C.; ARAÚJO, C.R.; TEIXEIRA, N.M. et al. Influência do meio e herança sobre os pesos ao nascer, aos 205 dias e aos 365 dias de idade de animais Nelore criados no nordeste do Brasil. Revista Brasileira de Zootecnia, v.22, n.3, p.455-465, 1993.

PAZ, C.C.P.; ALBUQUERQUE, L.G.; FRIES, L.A. Fatores de correção para ganho médio diáriono período do nascimento ao desmame em bovinos da raça Nelore. Revista Brasileira de Zootecnia, v.28, n.1, p.65-73, 1999.

PELICIONI, L.C.; PASCOA, L.; MUNIZ, A.S.D. et al. Efeito da idade da vaca ao parto e da data juliana de nascimento sobre características pré-desmama de bezerros da raça Gir. Revista Brasileira de Zootecnia, v.31, p.61-70, 2002.

PONS, S.B.; MILAGRES, J.C.; TEIXEIRA, N.M. Efeitos de fatores genéticos e de ambiente sobre o crescimento e o escore de conformação em bovinos Hereford no Rio Grande do Sul. I. Peso e escore de conformação à desmama. Revista Brasileira de Zootecnia, v.18, n.5, p.391-401, 1989.

STATISTICAL ANALYSES SYSTEM - SAS. SAS/STAT. User's guide. version 6.11. 4.ed. Cary: 1996. v.2. 842p.

TEIXEIRA, R.A. Heterose maternal e individual sobre o ganho de peso pré-desmama em bovinos Nelore $x$ Hereford e Nelore $x$ Angus. Jaboticabal: Universidade Estadual Paulista, 2000. 75p. Dissertação (Mestrado em Zootecnia) Universidade Estadual Paulista, 2000.

WOLF, P.G.L. Efeitos da heterose individual e materna sobre o ganho de peso do nascimento à desmama de bezerros Pampiano-Braford. Porto Alegre: Universidade Federal do Rio Grande do Sul, 1996. 114p. Dissertação (Mestrado em Ciências Veterinárias) - Universidade Federal do Rio Grande do Sul, 1996 